\title{
Online Detection of P300 and Error Potentials in a BCI Speller
}

\author{
Bernardo Dal Seno, ${ }^{1}$ Matteo Matteucci, ${ }^{1}$ and Luca Mainardi ${ }^{2}$ \\ ${ }^{1}$ IIT-Unit, Department of Electronics and Information, Politecnico di Milano, Piazza Leonardo da Vinci 32, 20133 Milano, Italy \\ ${ }^{2}$ IIT-Unit, Department of Bioengineering, Politecnico di Milano, Piazza Leonardo da Vinci 32, 20133 Milano, Italy
}

Correspondence should be addressed to Bernardo Dal Seno, bernardo.dalseno@polimi.it

Received 12 July 2009; Accepted 24 November 2009

Academic Editor: Sara L. Gonzalez

Copyright (C) 2010 Bernardo Dal Seno et al. This is an open access article distributed under the Creative Commons Attribution License, which permits unrestricted use, distribution, and reproduction in any medium, provided the original work is properly cited.

Error potentials (ErrPs), that is, alterations of the EEG traces related to the subject perception of erroneous responses, have been suggested to be an elegant way to recognize misinterpreted commands in brain-computer interface (BCI) systems. We implemented a P300-based BCI speller that uses a genetic algorithm (GA) to detect P300s, and added an automatic error-correction system (ECS) based on the single-sweep detection of ErrPs. The developed system was tested on-line on three subjects and here we report preliminary results. In two out of three subjects, the GA provided a good performance in detecting P300 (90\% and 60\% accuracy with 5 repetitions), and it was possible to detect ErrP with an accuracy (roughly 60\%) well above the chance level. In our knowledge, this is the first time that ErrP detection is performed on-line in a P300-based BCI. Preliminary results are encouraging, but further refinements are needed to improve performances.

\section{Introduction}

A brain-computer interface $(\mathrm{BCI})$ is an interface that does not entail muscle movements, but it bypasses any muscle or nerve mediation and connects a computer directly with the brain by picking up signals generated by the brain activity.

Among the different kinds of brain activity that can be used in a BCI, the $P 300$ phenomenon has been known [1] and investigated for many years. It is an event-related potential (ERP), traditionally described as a positive peak visible in an EEG recording at approximately $300 \mathrm{~ms}$ from an event. It follows unexpected, rare, or particularly informative stimuli, and it is typically stronger in the parietal area. The shape of the P300 depends on the characteristics of the stimuli and their presentation.

For BCI applications, the "exact" shape of the P300 is not so important as having a way to detect it. Detecting a P300 in a single trial is very difficult and, therefore, repeated stimuli are normally used to facilitate the selection of the one that has generated a P300. The number of repetitions can be predetermined for each user to get the best trade-off between speed and accuracy.

In [2], Donchin and colleagues presented the first P300based BCI, called also P300 speller, which permits to spell words. A grid of letters and symbols is presented to the user, and entire columns or rows are flashed one after the other in random order (see Figure 1 for an example). When the column/row containing the desired letter is flashed, a P300 is elicited. In Donchin's work, classification is made through stepwise discriminant analysis (SWDA) applied to averages of samples from epochs relative to the same stimulation (same row or same column).

Other BCI interfaces using the $\mathrm{P} 300$ protocol have been developed since then. In [3], a virtual-reality system is presented where subjects operate objects selected through the P300. Classification is made by comparing the correlation of single responses with the averages of all target and nontarget responses. In [4], subjects (healthy and impaired ones) control a cursor by choosing among four commands (up, down, left, right) via the P300. In this case, singlesweep detection is performed: independent component analysis (ICA) is used to decompose the EEG signal, a fuzzy classifier identifies a candidate P300 component among the ones extracted by ICA, and a neural network classifies it as target or nontarget. The system is more effective with healthy subjects, though no exact reason could be pinpointed. Finally, in [5], an initial attempt at using a BCI in a home environment is reported: a person with 
amyotrophic lateral sclerosis uses a P300 speller on a daily basis.

Another relevant event-related potential is the error potential (ErrP hereafter), which is generated when a subject makes a mistake, and, more interestingly for BCI applications, when the machine behaves differently from the user intent. Known since the late 1980s [6, 7], ErrPs were described as a negative shift in the electric potential over the fronto-central region (from $\mathrm{Fz}$ to $\mathrm{Cz}$ of the 1020 system) occurring 50-100 ms after an erroneous response (error negativity-Ne or error-related negativity-ERN) and a subsequent positive shift in the parietal region, whose maximum occurs between 200 and $500 \mathrm{~ms}$ after the error (error positivity-Pe). A high variability in shape, size, and delay of the Ne and Pe components has been observed as the effect of different underlying mechanism, whose nature is not yet certain [8].

In [9] the presence of ErrPs in a BCI paradigm (cursor movement by mu and beta rhythms) was revealed, as a positive peak at $\mathrm{Cz} 40 \mathrm{~ms}$ after the end of erroneous trials. This finding suggests an interesting application: the automatic detection of the errors made by a BCI in recognizing the user's intent and a way to improve its performances. Millán and colleagues $[10,11]$ made experiments with ErrPs found in a motor-imagery BCI. They trained a Gaussian classifier to automatically recognize ErrPs, reaching an accuracy of about $80 \%$.

In this work we present our experience in detecting P300 and ErrP in a P300-based speller with an integrated automatic error-correction system (ECS) based on the single-sweep ErrP detection.

\section{Experimental Setting}

We developed a classical BCI based on P300, the P300 speller, and integrated the use of ErrP in it. Our P300 speller is very similar in appearance and in functioning to the paradigm described by Donchin et al. [2]: 36 symbols are disposed on a $6 \times 6$ grid, and entire rows and columns of symbols are flashed one after the other in random order. The grid of symbols is visible in Figure 1. There are the letters from the alphabet, some digits, the space, and the backspace, represented as BS in the right bottom corner. The intensification of rows and columns lasts for $125 \mathrm{~ms}$ and the matrix remains blank for $125 \mathrm{~ms}$ between two consecutive flashes. Each row and column is flashed exactly once in the first 12 stimulations (a block of 12 consecutive stimulations is called a repetition); then another round of 12 stimulations is repeated, with flashing of rows and columns done in a new random order. We used 5 repetitions with no pause between repetitions. Please note that the number of repetitions is lower than usual. The choice is instrumental to stress the system under an unfavorable situation, thus soliciting a substantial number of misspelled letters.

After the fifth repetition, the P300 system detects the row and the column that are more likely to have elicited a P300, and selects the letter at their intersection. After a pause of $1 \mathrm{~s}$, the letter is presented to the user in a big rectangle that pops up in front of the grid (see Figure 1). The presentation of the

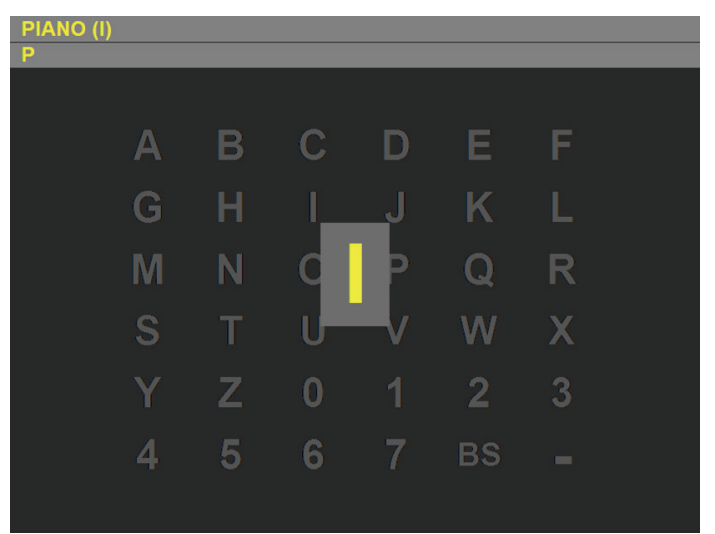

Figure 1: Graphical interfaces of the P300 spellers used in the experiments, showing the moment of the letter feedback used for ErrP-based confirmation.

letter should elicit an ErrP if the letter predicted by the P300 system is different from the one the user intended.

An ErrP detection system figures out if any ErrP is elicited by the presentation of the selected letter, and in that case it overrides the P300 speller and cancels the last selection; otherwise, the letter is appended to the text at the top. After a 2-3 s pause (this parameter is tuned to each subject's requirements), the speller starts a new series of stimulations for the next letter. A trial, in this context, is the whole series of 60 row/column flashes (12 flashes times 5 repetitions) together with the feedback of the speller selection made for each letter, that is, a single trial is composed of 60 P300 stimulations and 1 ErrP stimulation (a trial is about $15 \mathrm{~s}$ long).

In the online experiments, the users interact with the speller in two ways: in copy mode they are asked to select letters indicated by the BCI before each trial, so as to simplify the evaluation of the performance; in free mode subjects spell words of their own choosing. In copy mode, the system performs one trial for each letter, and it goes on to the next letter even when the P300 classifier is wrong; in this mode the ErrP correction system is not active. In free mode the ErrP correction system is active, and the user has to hit the backspace to correct a misspelled letter only when the error is not automatically recognized. During training, the speller is used in copy mode only. The GA and ErrP training was performed sequentially. In the ErrP training, in order to elicit error-related responses, the letter feedback is chosen wrongly in $20 \%$ of the times and correctly in $80 \%$ of the cases.

The speller we have implemented is based on BCI2000 [12], a general-purpose software system developed at the Wadsworth Center of the New York State Department of Health in Albany, New York, USA, for brain-computer interface (BCI) research. We developed three main components: a source module that acquires EEG data from our amplifier, an application derived from the built-in P300 speller, and a dual-classifier processing module to handle both P300 and ErrP classification. The application module implements the P300 speller with ErrP-based error correction, as described above, and a precise synchronization system (fully described 
in [13]). The processing module splits the EEG signals in epochs synchronized on the stimulation instants, processes the data, and performs the classification of the epochs according to two separate processing chains, one for P300s and one for ErrPs, briefly described below.

\section{Data Processing}

EEG data are acquired with an EBNeuro BE Light amplifier at locations $\mathrm{Fz}, \mathrm{Cz}, \mathrm{Pz}$, and $\mathrm{Oz}$, and at a frequency of $512 \mathrm{~Hz}$. Also, EOG is recorded from the right eye of the subject. EOG is not used for classification, but it is used to discard noisy epochs during training and is kept for future analysis. For P300 detection, a logistic classifier [14] is used, trained on features extracted through a genetic algorithm.

Genetic algorithms are a class of optimization algorithms that mimic the way natural evolution works. In a genetic algorithm, a set of possible solutions to an optimization problem are coded in strings called chromosomes; solutions are evaluated, and the best ones (those with highest fitness) are selected and combined together to form new possible solutions, in a process that mimics evolution among living beings. After some repetitions of the procedure, good solutions emerge.

In the genetic algorithm used in this work, each individual (chromosome) represents a set of possible features for discriminating the presence of a P300 in EEG recordings. Each gene encodes a feature and an EEG channel from which to extract it; a feature is obtained by multiplying the EEG channel by a weight function, whose exact shape is encoded by parameters in genes (see Figure 2 for examples of weight functions). Genetic operators are a variant of 1-point crossover and uniform mutation, and tournament selection with elitism is used. The fitness of a chromosome is the 4 -fold cross-validated performance obtained by training a logistic classifier on the encoded features extracted from the training set. For a complete description of the algorithm, please see [15].

An analysis of the combination of the features extracted by the genetic algorithm and the classifier trained on the training set allows to compute weights assigned to individual EEG samples. In this way, the resulting classifier is very fast to apply online.

For ErrP detection, a simpler method is used, also because fewer training data are available (there is one ErrP stimulation per letter versus sixty for P300).

EEG data are segmented in epochs, whose extremes are found with the algorithm explained below. Epochs are then filtered in the band $1-10 \mathrm{~Hz}$ to improve the signal-to-noise ratio by eliminating frequency components extraneous to ErrPs. EEG samples are fed into a classifier trained through linear discriminant analysis (LDA).

On average, a difference between ErrP and non-ErrP epochs is observable only in some intervals of the segmented epoch, and these intervals depend on the subject. For these reasons we developed a way to automatically determine significant intervals in the ErrP for classification from the training data. Training data are first segmented in epochs

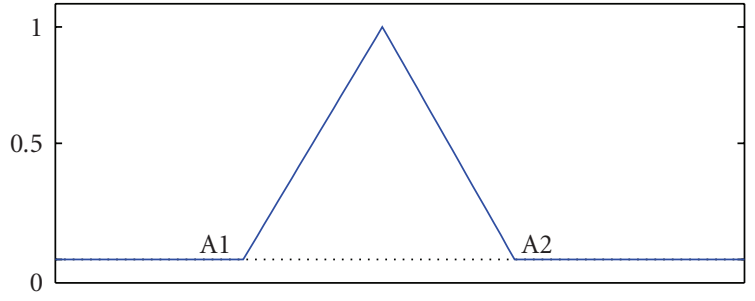

(a)

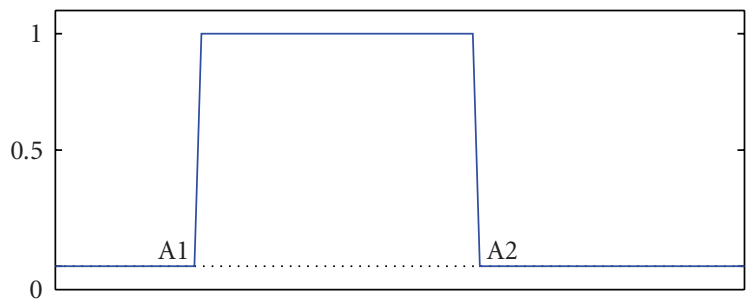

(b)

Figure 2: Weight functions used for feature extraction.

ranging from $100 \mathrm{~ms}$ before the stimulation instant (feedback onset) to $500 \mathrm{~ms}$ after it. Epochs containing strong EOG activity ( $>100 \mu \mathrm{V}$ at any point) are automatically discarded before further analysis. A $1-10 \mathrm{~Hz}$ pass-band filter is then applied. For each channel $c$ and time point $t$, the signals $s_{c, 1}(t)$ from ErrP epochs and $s_{c, 0}(t)$ from non-ErrP epochs can be viewed as two sets of random variables. A $t$-test is used to check if, for any given $t$ and $c$, the mean of $s_{c, 1}(t)$ differs significantly from the mean of $s_{c, 0}(t)$; the significance level has been chosen to be 0.01 , but much smaller $P$-values have been often found in analyzing data. The $t$-test is used only to find a time interval to use for classification, so the validity of its assumptions (Gaussian distributions with equal variance) is not very important; nevertheless, we ran some statistical tests on the data and they were satisfied.

The points detected by the $t$-test tend to lie in groups, because the filtered signals have a strong autocorrelation for short lags. However, many intervals of different sizes, with "holes" in between (see the top part of Figure 3 for an example), are usually detected, while we are interested only in finding one contiguous time interval containing all the interesting features of signals. We used DBSCAN [16], a clustering algorithm based on density, to fill holes and discard isolated points or small intervals. The biggest interval is selected and used for classification.

The training phase produces both a time interval and a linear classifier. During online classification, the procedure is very fast. EEG epochs are cut according to the interval found, and the classifier is applied to pass-band filtered epochs.

\section{Results}

Three subjects participated in a first set of online experiments. The P300 speller used 5 repetitions of each stimulation per letter; for Subject B1 we had to reduce the number of repetitions to 4 in order to have a reasonable 


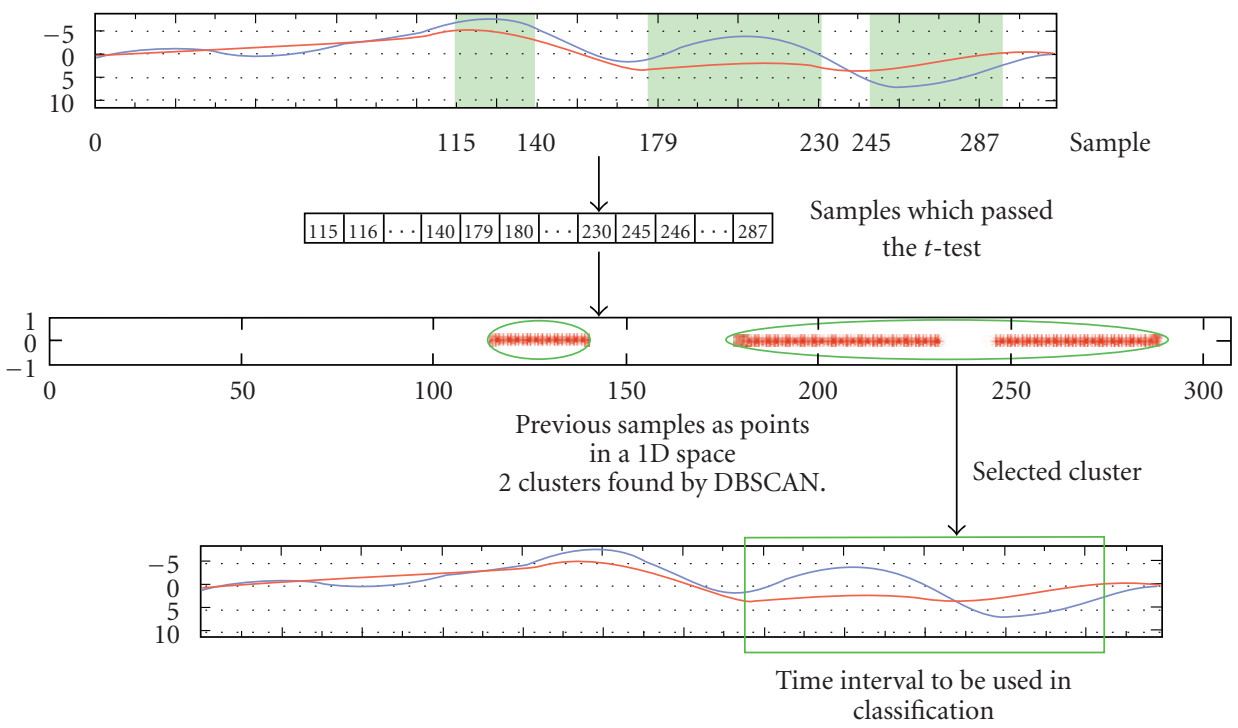

Figure 3: Procedure for the identification of significant intervals. Top: shadowed areas contain the samples that passed the $t$-test with a $P$-value of .01 or less. Middle: clustering of samples. Bottom: the interval used for classification.

TABLE 1: Results of the GA online in free mode. Training set size is the number of letters spelled in copy mode to collect training examples for the GA classifier. Performance is given as the number of correctly predicted letters over the total numbers of letters in the online usage.

\begin{tabular}{lccc}
\hline Subject & $\begin{array}{c}\text { Training } \\
\text { set size }\end{array}$ & $\begin{array}{c}\text { No. } \\
\text { repetitions }\end{array}$ & $\begin{array}{c}\text { online } \\
\text { performance }\end{array}$ \\
\hline B1 & 196 & 4 & $74 / 109(68 \%)$ \\
B3 & 108 & 5 & $137 / 202(68 \%)$ \\
\hline
\end{tabular}

number of errors (the online performance in copy mode for Subject B1 was $90 \%$ with 5 repetitions). On the other hand, Subject B2 had a poor performance mainly due to low concentration; the subject reported problems in focusing on the task, probably because of a failure of the brightness regulation of the computer screen that affected the online recordings. All results confirm the offline figures reported in [15], and confirm the validity of the GA-based classification method.

Subjects B1 and B3 also used the BCI to spell words in free mode, where the error correction mechanism was enabled. The results are shown in Table 1 and confirm that the classifier found by the GA can be used to really drive a BCI application. Subject B2 could have tried to use the speller by increasing the number of repetitions, but as the data was recorded also to evaluate error potentials, this would have made the recording sessions much longer.

Results of the online experiments are shown in Table 2. The classifiers were tested in sessions different from those used for training, so they are really indicative of a possible online use. For both users the classification performance is well above chance level, but this is not enough to say whether ErrP detection has been useful for such users. To test it, we computed the gain obtained by the inclusion
TABLe 2: Results of the online ErrP classification. Training size is the number of letters for each class from the ErrP copy mode session. Performance is the fraction of correct classification in the free mode experiment.

\begin{tabular}{cccc}
\hline & & Train. & online \\
\multicolumn{2}{c}{ Subject } & Size & performance \\
\hline B1 & ErrP & 84 & $23 / 35(66 \%)$ \\
& N-ErrP & 290 & $51 / 74(69 \%)$ \\
B3 & ErrP & 65 & $38 / 65(58 \%)$ \\
& N-ErrP & 193 & $91 / 137(66 \%)$ \\
\hline
\end{tabular}

of an automatic ErrP correction system. The gain is based on the computation of the Utility metric we recently proposed (see [17] for details): for subject B1 we obtained a small improvement (gain $=1.0011$ ), while in subject B3 a detriment to the performance is observed (gain $=0.8733$ ).

\section{Discussion}

In this paper we have presented an experiment that-to the best of our knowledge-is the first attempt to use a P300 BCI with an integrated error-correction mechanism based on ErrPs. Although the number of subjects participating to the online study is quite limited, results are encouraging and confirm the feasibility of ErrP single-sweep detection already verified in more populated offline studies such as [18] or [13].

The use of a genetic algorithm for the definition of features to be used in P300 detection has proven its strength also in the online use, after good results in offline analysis [15]. In principle, the very same algorithm could be used for the ErrP feature design, but this is somehow prevented by the reduced number of examples that can be gathered in training sessions. A different strategy could be devised to collect ErrP 
examples automatically during the use of our P300-based BCI application. Each back space in free mode can be treated as an explicit tagging of an ErrP by the user. With this strategy, data gathering would be still time consuming (we are not changing the odds for ErrP elicitation after all), but it could be more acceptable by the user, and it might enhance her experience with the speller as time passes.

The results presented are encouraging, but some additional work is still needed to improve the performance. In particular, it is important that ErrP detection reaches a high accuracy, higher than P300 detection. The reason is that ErrP stimulations are generated only once after each letter selection, and this is the only chance to detect an ErrP. An accuracy higher than chance is not sufficient to have a usable interface or a significant improvement as documented by the measured gain. In addition, to make the inclusion of ErrP corrections more profitable, the performances of the ErrP classifier should be tuned on a user-by-user basis. This could be done by maximizing the above mentioned gain. This was not done in the present paper, where we did not tune the ErrP classifier in favor of false positives (nor false negatives [19]).

In addition, to make the system more useful in practice, we plan to refine our processing methods and presentation interface (to better capture the subject attention) so as to increase the performance of the ErrP classifier; in a more extensive study with more subjects we will assess the online performance of the enhancement.

\section{Acknowledgments}

This work has been partially supported by the Italian Institute of Technology (IIT), and by the grant "Brain-Computer Interfaces in Everyday Applications" from Politecnico di Milano and Regione Lombardia.

\section{References}

[1] S. Sutton, M. Braren, J. Zubin, and E. R. John, "Evokedpotential correlates of stimulus uncertainty," Science, vol. 150, no. 3700, pp. 1187-1188, 1965.

[2] E. Donchin, K. M. Spencer, and R. Wijesinghe, "The mental prosthesis: assessing the speed of a P300-based braincomputer interface," IEEE Transactions on Rehabilitation Engineering, vol. 8, no. 2, pp. 174-179, 2000.

[3] J. D. Bayliss, S. A. Inverso, and A. Tentler, "Changing the P300 brain computer interface," Cyberpsychology \& Behavior, vol. 7, no. 6, pp. 694-704, 2004.

[4] F. Piccione, F. Giorgi, P. Tonin, et al., "P300-based brain computer interface: reliability and performance in healthy and paralysed participants," Clinical Neurophysiology, vol. 117, no. 3, pp. 531-537, 2006.

[5] T. M. Vaughan, D. J. McFarland, G. Schalk, et al., "The wadsworth BCI research and development program: at home with BCI," IEEE Transactions on Neural Systems and Rehabilitation Engineering, vol. 14, no. 2, pp. 229-233, 2006.

[6] M. Falkenstein, J. Hohnsbein, J. Hoormann, and L. Blanke, "Effects of crossmodal divided attention on late ERP components. II. Error processing in choice reaction tasks," Electroencephalography and Clinical Neurophysiology, vol. 78, no. 6, pp. 447-455, 1991.
[7] W. J. Gehring, B. Goss, M. G. H. Coles, D. E. Meyer, and E. Donchin, "A neural system for error detection and compensation," Psychological Science, vol. 4, no. 6, pp. 385390, 1993.

[8] M. Falkenstein, "ERP correlates of erroneous performance," in Errors, Conflicts, and the Brain: Current Opinions on Performance Monitoring, M. Ullsperger and M. Falkenstein, Eds., pp. 5-14, 2004.

[9] G. Schalk, J. R. Wolpaw, D. J. McFarland, and G. Pfurtscheller, "EEG-based communication: presence of an error potential," Clinical Neurophysiology, vol. 111, no. 12, pp. 2138-2144, 2000.

[10] P. W. Ferrez and J. del R. Millán, "You are wrong!automatic detection of interaction errors from brain waves," in Proceedings of the 19th International Joint Conference on Artificial Intelligence (IJCAI '05), pp. 1413-1418, Edinburgh, UK, August 2005.

[11] P. W. Ferrez and J. del R. Millán, "Simultaneous real-time detection of motor imagery and error-related potentials for improved BCI accuracy," in Proceedings of the 4th International Brain-Computer Interface Workshop \& Training Course, pp. 197-202, Graz, Austria, September 2008.

[12] J. Mellinger and G. Schalk, "BCI2000: a general-purpose software platform for BCI research," in Towards Brain-Computer Interfacing, G. Dornhege, J. del R. Millán, T. Hinterberger, D. J. McFarland, and K.-R. Müller, Eds., MIT Press, Cambridge, Mass, USA, 2007.

[13] B. Dal Seno, Toward an integrated P300- and ErrP-based braincomputer interface, Ph.D. thesis, Politecnico di Milano, Milan, Italy, 2009.

[14] S. le Cessie and J. C. van Houwelingen, "Ridge estimators in logistic regression,” Applied Statistics, vol. 41, no. 1, pp. 191201, 1992.

[15] B. Dal Seno, M. Matteucci, and L. Mainardi, "A genetic algorithm for automatic feature extraction in P300 detection," in Proceedings of the International Joint Conference on Neural Networks (IJCNN '08), pp. 3145-3152, Hong Kong, 2008.

[16] M. Ester, H.-P. Kriegel, J. Sander, and X.-A. Xu, "A densitybased algorithm for discovering clusters in large spatial databases with noise," in Proceedings of the 2nd International Conference on Knowledge Discovery and Data Mining (KDD '96), pp. 226-231, AAAI Press, Portland, Ore, USA, August 1996.

[17] B. Dal Seno, M. Matteucci, and L. Mainardi, "The utility metric: a novel method to assess the overall performance of discrete brain-computer interfaces," IEEE Transactions on Neural Systems and Rehabilitation Engineering. In press.

[18] G. Visconti, B. Dal Seno, M. Matteucci, and L. Mainardi, "Automatic recognition of error potentials in a P300-based brain-computer interface," in Proceedings of the 4th International Brain-Computer Interface Workshop \& Training Course, pp. 238-243, Graz, Austria, September 2008.

[19] B. Blankertz, G. Dornhege, R. Krepki, et al., "Boosting bit rates and error detection for the classification of fast-paced motor commands based on single-trial EEG analysis," IEEE Transactions on Neural Systems and Rehabilitation Engineering, vol. 11, no. 2, pp. 127-131, 2003. 

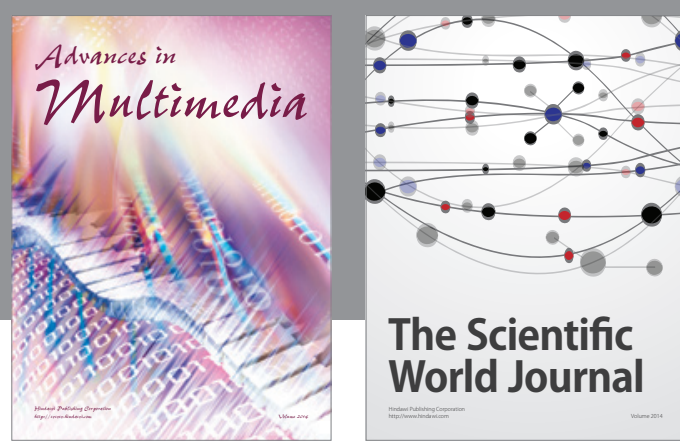

The Scientific World Journal
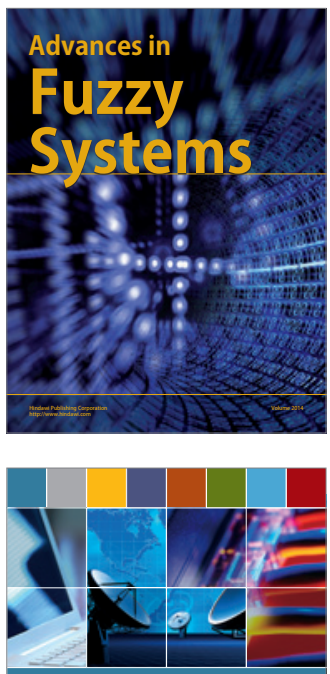

Computer Networks and Communications
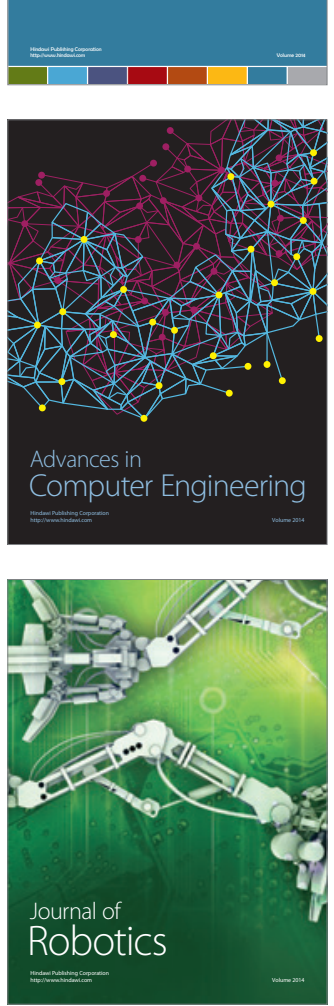
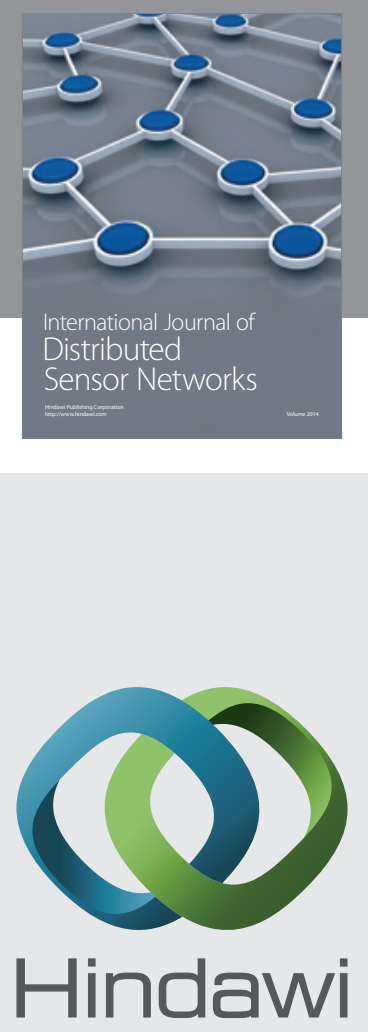

Submit your manuscripts at

http://www.hindawi.com
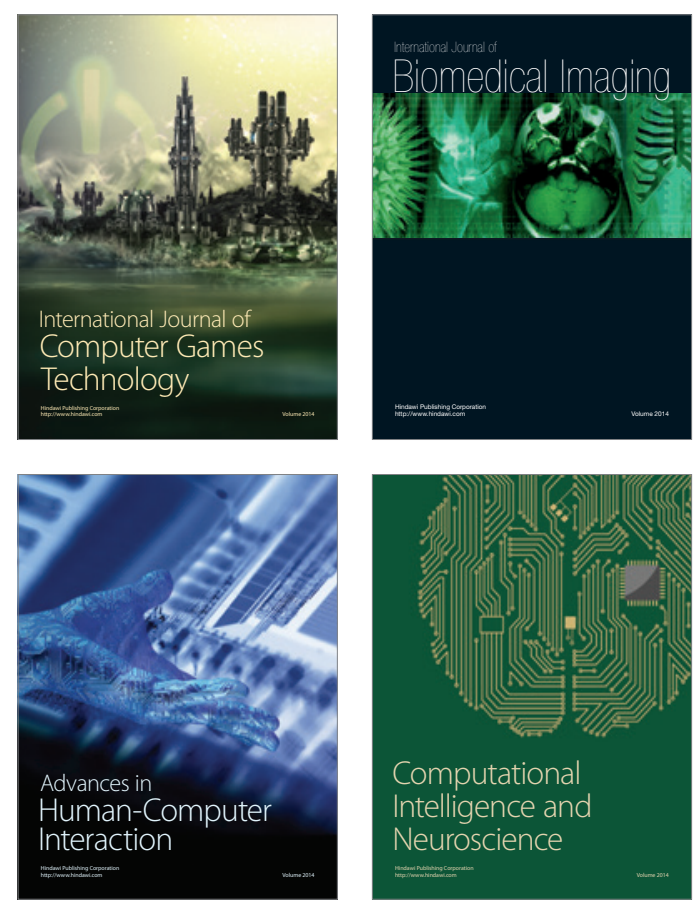
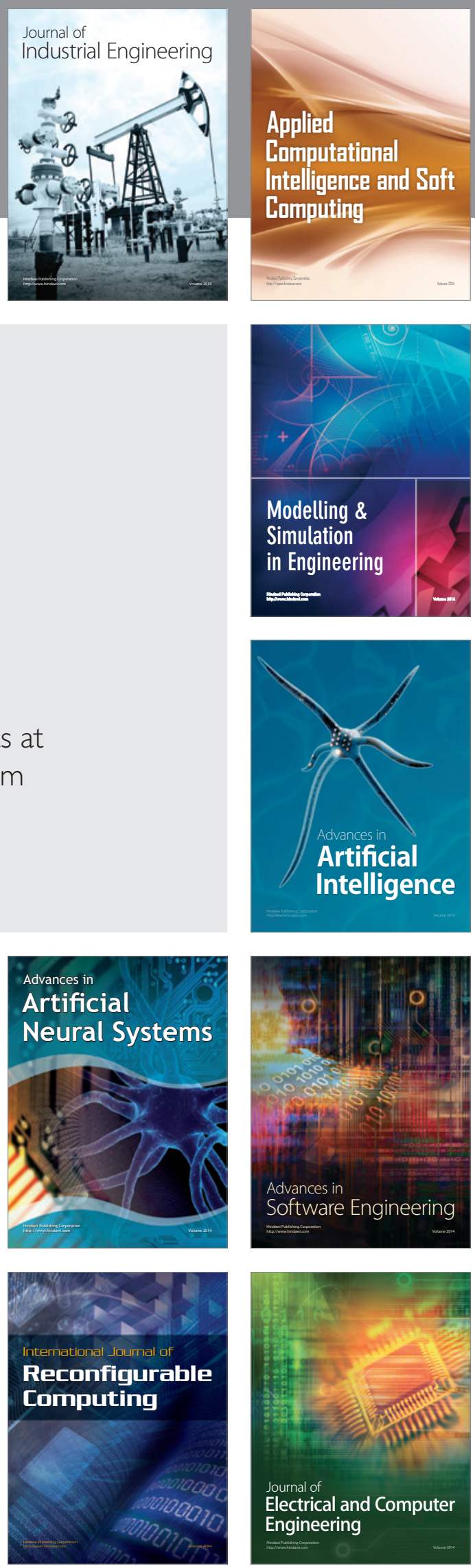